\title{
THE NATURAL MEASURE OF NONATTRACTING CHAOTIC SETS AND ITS REPRESENTATION BY UNSTABLE PERIODIC ORBITS
}

\author{
MUKESHWAR DHAMALA \\ School of Physics, Georgia Institute of Technology, Atlanta, GA 30332, \\ School of Medicine, Emory University, Atlanta, GA 30322, USA \\ YING-CHENG LAI \\ Departments of Mathematics and Electrical Engineering, \\ Systems Science and Engineering Research Center, \\ Arizona State University, Tempe, AZ 85287, USA
}

Received October 10, 2001; Revised November 2, 2001

\begin{abstract}
The natural measure of a chaotic set in a phase-space region can be related to the dynamical properties of all unstable periodic orbits embedded in the chaotic set contained in that region. This result has been shown to be valid for hyperbolic chaotic invariant sets. The aim of this paper is to examine whether this result applies to nonhyperbolic, nonattracting chaotic saddles which lead to transient chaos in physical systems. In particular, we examine, quantitatively, the closeness of the natural measure obtained from a long trajectory on the chaotic saddle to that evaluated from unstable periodic orbits embedded in the set. We also analyze the difference between the long-time average values of physical quantities evaluated with respect to a dense trajectory and those computed from unstable periodic orbits. Results with both the Hénon map and the Ikeda-Hammel-Jones-Moloney map for which periodic orbits can be enumerated lend credence to the conjecture that the unstable periodic-orbit theory of the natural measure is applicable to nonhyperbolic chaotic saddles.
\end{abstract}

Keywords: Transient chaos; unstable periodic orbits; natural measure; ergodic averages; nonhyperbolicity.

\section{Introduction}

Chaotic saddles are nonattracting dynamical invariant sets in the phase space of nonlinear systems [Grebogi et al., 1983; Kantz \& Grassberger, 1985; Tél, 1990, 1996]. A trajectory starting from a random initial condition in a phase-space region containing a chaotic saddle typically stays near the saddle, exhibiting a chaotic-like dynamics for a finite amount of time before exiting the region eventually and approaching asymptoticaly to a final state that is usually not chaotic. Chaos in this case is only transient. Physically, chaotic saddles lead to observable phenomena such as chaotic scat- tering [Focus Issue, 1993], fractal basin boundaries [McDonald et al., 1985], fractal concentrations of passive particle advected in open hydrodynamical flows [Eckhardt \& Aref, 1988; Jung et al., 1993; Ziemniak et al., 1994; Péntek et al., 1995a; Péntek et al., 1995b; Péntek et al., 1995c; Neufeld \& Tél, 1995] and fractal distribution of chemicals in environmental flows [Toroczkai et al., 1998]. Mathematically, chaotic saddles are closed, bounded, and invariant sets having dense orbits. They are the soul of chaotic dynamics [Smale, 1967].

In a nonlinear system, there are typically two situations where transient chaos can occur. The first is in parameter regimes after crises [Grebogi 
et al., 1983]. At a crisis, a chaotic attractor collides with its own basin boundary and is converted into a chaotic saddle [Grebogi et al., 1983]. After the crisis, the main dynamical invariant sets are chaotic saddles [Lai et al., 1993]. The second case where chaotic saddles arise corresponds to periodic windows created at saddle-node bifurcations. In a given periodic window, one finds the coexistence of a chaotic saddle and an attracting periodic orbit. The attracting orbit bifurcates in an infinite period-doubling cascade leading to a small chaotic attractor. The collision of this small chaotic attractor with the coexisting chaotic saddle marks the end of the window and the recovery of the large chaotic attractor in an interior crisis [Grebogi et al., 1983; Szabó et al., 1996; Szabó et al., 2000].

In the study of transient chaos, one often computes dynamical invariants associated with the chaotic saddle. They can be, for instance, the Lyapunov exponents, the fractal dimension, the topological entropy, and other invariants of the probability density or measure. These invariants determine completely the behavior of physical observables such as the scattering function in chaotic scattering [Focus Issue, 1993], the probability for a trajectory to approach a specific final state in the presence of fractal basin boundaries [McDonald et al., 1985], and the chemical reaction rate in environmental flows [Toroczkai et al., 1998]. The dynamical invariants associated with any chaotic invariant set are physically meaningful only when the measure being considered is the natural measure. For a chaotic attractor, the natural measure is invariant under the time evolution of the dynamics, and it can be obtained by using typical trajectories on the attractors, that is, by trajectories originated from randomly chosen initial conditions in the basin of attraction. For a nonattracting chaotic saddle, the natural measure can be computed by monitoring an ensemble of typical trajectories on the saddle (see Sec. 2 for a formal definition of the natural measure). To better understand and manipulate transient chaos, it is thus of physical interest to be able to understand and to characterize the natural measure of a chaotic saddle in terms of fundamental dynamical quantities. The most fundamental building blocks of a chaotic dynamics are the infinite number of unstable periodic orbits embedded in the chaotic invariant set [Morita et al., 1987; Gunaratne \& Procaccia, 1987; Auerbach et al., 1988a; Auerbach et al., 1988b; Auerbach, 1990; Cvitanović, 1992; Christiansen et al., 1997].

A key contribution along these lines was made in [Grebogi et al., 1988] in which the authors obtained an expression for the invariant natural measure in terms of the magnitude of the eigenvalues of the unstable periodic orbits embedded in the chaotic set. They proved [Grebogi et al., 1988] the correctness of their expression but only for the special case of an hyperbolic dynamics. ${ }^{1}$ The validity of their results for physical, which are typically nonhyperbolic, situations remained, however, only a conjecture. Recently, it was numerically verified that this quantification of the natural measure by unstable periodic orbits was valid for nonhyperbolic chaotic attractors [Lai et al., 1997; Lai, 1997]. The purpose of this paper is to provide evidences for the applicability of the results of [Grebogi et al., 1988] to nonhyperbolic chaotic saddles that occur commonly in nonlinear systems [Lai et al., 1993]. Because of the difficulty of enumerating unstable periodic orbits in high dimensions, we restrict our study to two-dimensional maps or, equivalently, three-dimensional flows. In order to show that the results by Grebogi et al. [1988] are also valid for the case of nonhyperbolic chaotic saddles, we take two approaches: (1) we compare the natural measure computed from a dense orbit on a chaotic saddle with that computed from unstable periodic orbits embedded in the saddle; and (2) we compare the average physical quantities computed from a dense orbit with those computed from the periodic orbits. Results with the Hénon map and the IkedaHammel-Jones-Moloney (IHJM) map for which periodic orbits can be enumerated lend credence to the conjecture that the unstable periodic-orbit theory of the natural measure is applicable to nonhyperbolic chaotic saddles, which are an important type of chaotic invariant sets of great physical interest. A brief account of part of the numerical results has been published [Dhamala et al., 1999].

\footnotetext{
${ }^{1}$ The dynamics is hyperbolic on a chaotic set if at each point of the trajectory the tangent space can be split into an expanding and a contracting subspace and the angle between them is bounded away from zero. Furthermore, the expanding subspace evolves into the expanding one along the trajectory and the same is true for the contracting subspace. Otherwise the set is nonhyperbolic. In general, nonhyperbolicity is a complicating feature because it can cause fundamental difficulties in the study of the chaotic systems, a known one being the shadowability of numerical trajectories by real ones [Hammel et al., 1987; Hammel et al., 1988; Grebogi et al., 1990; Dawson et al., 1994].
} 
The rest of the paper is organized as follows. In Sec. 2, we give the definition of the natural measure for chaotic saddles and describe the unstable periodic orbit theory for transient chaos [Grebogi et al., 1988]. In Sec. 3, we provide numerical evidence for the validity of the theory for nonhyperbolic chaotic saddles. For completeness, in Appendix we describe our algorithm to compute complete sets of unstable periodic orbits [Davidchack \& Lai, 1999; Davidchack et al., 2001]. Discussions are presented in Sec. 4.

\section{Natural Measure of Chaotic Saddles and Its Characterization by Unstable Periodic Orbits}

\subsection{Definition of natural measure of chaotic saddles}

We consider dynamical systems described by twodimensional invertible maps, $\mathbf{x}_{n+1}=\mathbf{M}\left(\mathbf{x}_{n}\right)$, where $\mathbf{x} \in \mathbf{R}^{2}$. These maps arise from the Poincaré surface of section of three-dimensional flows. Imagine a phase-space region $S$ that contains a nonattracting chaotic saddle $\Sigma$. The stable and the unstable manifolds of the chaotic saddle are sets of points that approach to it asymptotically under the forward and backward iterations of the map, respectively. If a large number $N_{0}$ of random initial conditions are distributed in $S$, the corresponding trajectories will leave $S$ eventually. They do so by being attracted along the stable manifold, wandering near the chaotic saddle for a finite amount of time, and then exiting along the unstable manifold. Let $N(n)$ be the number of trajectories that still remain in $S$ at time $n$. Due to the chaotic nature of the saddle, for large $n, N(n)$ decreases exponentially in time (typical for dissipative systems),

$$
N(n)=N_{0} e^{-n / \tau}
$$

where $\tau$ is the average lifetime of the chaotic transients, and the inverse of which, $\kappa \equiv 1 / \tau$, is the escape rate of the saddle [Tél, 1990, 1996]. More formally, the lifetime is defined to be

$$
\frac{1}{\tau}=\lim _{n \rightarrow+\infty} \lim _{N_{0} \rightarrow \infty} n^{-1} \ln \left[\frac{N(n)}{N_{0}}\right] .
$$

Since trajectories escape from the chaotic saddle along the unstable manifold, at large positive time $n$, the $N(n)$ trajectory points will be in the vicinity of the unstable manifold. Let $C$ be a small box within $S$ that contains part of the unstable manifold. The natural measure associated with the unstable manifold in $C$ can thus be defined as [Grebogi et al., 1988; Hsu et al., 1988]

$$
\mu_{u}(C)=\lim _{n \rightarrow+\infty} \lim _{N_{0} \rightarrow \infty} \frac{N_{u}(n, C)}{N(n)},
$$

where $N_{u}(n, C)$ is the number of the $N(n)$ orbits in $C$ at time $n$. Similarly, the natural measure of the stable manifold in a box $C$ in $S$ can be defined as [Grebogi et al., 1988; Hsu et al., 1988]

$$
\mu_{s}(C)=\lim _{n \rightarrow+\infty} \lim _{N_{0} \rightarrow \infty} \frac{N_{s}(n, C)}{N(n)},
$$

where $N_{s}(n, C)$ is the number of initial conditions in $C$ whose trajectories do not leave $S$ before time $n$.

From the definitions (3) and (4), we see that the natural measures associated with the stable and the unstable manifolds in $C$ are determined by the number of trajectory points in $C$ at time zero and time $n$, respectively. The natural measure of the chaotic saddle, $\mu$, can then be defined by considering $N_{m}(\rho, n, C)$, the number of trajectory points in $C$ at a time $\rho n$ in between zero and $n$,

$$
\mu(C)=\lim _{n \rightarrow+\infty} \lim _{N_{0} \rightarrow \infty} \frac{N_{m}(\rho, n, C)}{N(n)},
$$

where $0<\rho<1, N_{m}(0, n, C)=N_{s}(n, C)$, and $N_{m}(1, n, C)=N_{u}(n, C)$. For large $N_{0}$ and $n$, trajectories remaining in $S$ would stay near the chaotic saddle for most of the time between zero and $n$, except at the beginning when they are attracted towards the saddle along the stable manifold, and at the end when they are exiting along the unstable manifold. Thus, the measure defined in Eq. (5) is independent of $\rho$, insofar as $0<\rho<1$.

Note that, although $N(n)$ decreases exponentially in time, this decaying factor has been compensated in the definitions of the natural measures (3-5). These measures are thus invariant under the dynamics, and they are also called the conditionally invariant measures [Tél, 1990, 1996]. Numerically, the natural measure of the chaotic saddle can be computed by using the sprinkler method [Hsu et al., 1988] or the PIM-triple method [Nusse et al., 1989; Jacobs et al., 1997], the latter can generate long trajectories on the chaotic saddle. Dynamical invariants of the chaotic saddle, such as the fractal dimensions and the Lyapunov exponents, can then be defined with respect to the conditionally invariant measure of the saddle. 


\subsection{Unstable-periodic-orbit characterization}

To relate the natural measure to unstable periodic orbits embedded in the saddle [Grebogi et al., 1988], we cover the chaotic set with a grid of partitioning cells, each being confined by segments of the stable and unstable manifolds. If the cells are small compared with the size of the phase-space region in which the chaotic set lies, each cell can be regarded as being rectangular, as shown in the upper panel of Fig. 1, where the horizontal and vertical sides are segments of the stable and unstable manifolds, respectively. Denote this cell by $C_{i}$. Now imagine that a large number $N_{0}$ of initial conditions is chosen according to the natural measure of the chaotic saddle. The measure of the unstable manifold $\mu_{u}$ contained in the cell $C_{i}$ is the fraction of trajectories that are still in $C_{i}$ in the limit $n \rightarrow \infty$. Let $\mathbf{x}_{0}$ be an initial condition on the part of the chaotic saddle in the cell $C_{i}$. Due to recurrence or ergodicity of trajectories on chaotic saddles, the trajectory from $\mathbf{x}_{0}$ comes back to some point $\mathbf{x}_{p}$ in $C_{i}$, say, after $p$ iterations, as shown in Fig. 1(a). Let $a b$ be the horizontal line segment through $\mathbf{x}_{0}$ ending at the two unstable-manifold segments, and $c^{\prime} d^{\prime}$ be the vertical line segment through $\mathbf{x}_{p}$ ending at the two stable-manifold segments, as shown in the lower panel of Fig. 1. Since $a b$ is parallel to the stable-manifold segments and, since $\mathbf{x}_{0}$ maps to $\mathbf{x}_{p}$ after $p$ iterations, the image of $a b$ under the $p$ times iterated map $\mathbf{M}^{p}(\mathbf{x})$ is a shorter horizontal line segment $a^{\prime} b^{\prime}$ straddling $\mathbf{x}_{p}$. Similarly, the $p$ th

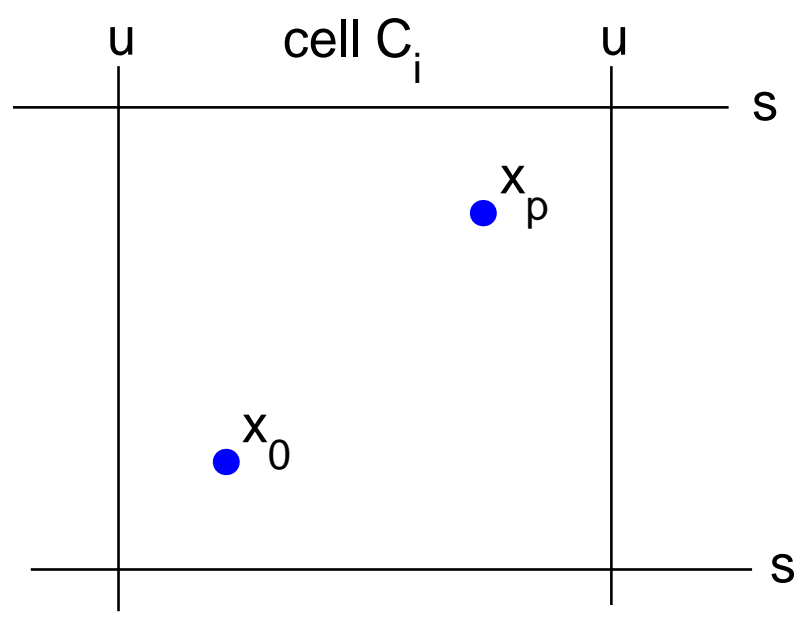

(a) preimage of $c^{\prime} d^{\prime}$ is a shorter vertical line segment $c d$ straddling $\mathbf{x}_{0}$. Now construct two rectangles efgh and $e^{\prime} f^{\prime} g^{\prime} h^{\prime}$ with side lengths $(a b, c d)$ and $\left(a^{\prime} b^{\prime}, c^{\prime} d^{\prime}\right)$, respectively. We see that the rectangle efgh maps to the rectangle $e^{\prime} f^{\prime} g^{\prime} h^{\prime}$ under $\mathbf{M}^{p}(\mathbf{x})$. Since both rectangles have an overlapping region, and since the dynamics is contracting in the horizontal direction and expanding in the vertical direction, there must be at least one point in the overlapping region whose location is not influenced by the action of the $p$ thiterated map $\mathbf{M}^{p}(\mathbf{x})$. That is, there must be an unstable fixed point $\mathbf{x}_{i p}$ of $\mathbf{M}^{p}(\mathbf{x})$ in the overlapping region in the cell $C_{i}$.

To estimate the contribution to $\mu$ from the fixed point $\mathbf{x}_{i p}$, we assume that $c^{\prime} d^{\prime}$ has a length $\varepsilon$. Thus, we have $\varepsilon / L_{1}\left(\mathbf{x}_{i p}\right)$ for the length of $c d$, where $L_{1}\left(\mathbf{x}_{i p}\right)$ is the unstable (expanding) eigenvalue of the fixed point $\mathbf{x}_{i p}$. Since the natural measure is uniform along the unstable direction and since there are only about $e^{-p / \tau}$ of the total number of trajectories that are still in $S$ at time $p$, we see that, associated with the unstable fixed point $\mathbf{x}_{i p}$, the fraction of trajectories that are still in $C_{i}$ in $p$ iterations is

$$
\frac{\left[\varepsilon / L_{1}\left(\mathbf{x}_{i p}\right)\right] e^{-p / \tau}}{\varepsilon e^{-p / \tau}}=\frac{1}{L_{1}\left(\mathbf{x}_{i p}\right)}
$$

Let $C \in S$ be a subregion in the phase space that contains part of the chaotic saddle, and let $n>p$ be a time at which we wish to examine how many trajectory points, out of those from the $N_{0}$ initial conditions, still remain in $C$. From Eq. (6), we see

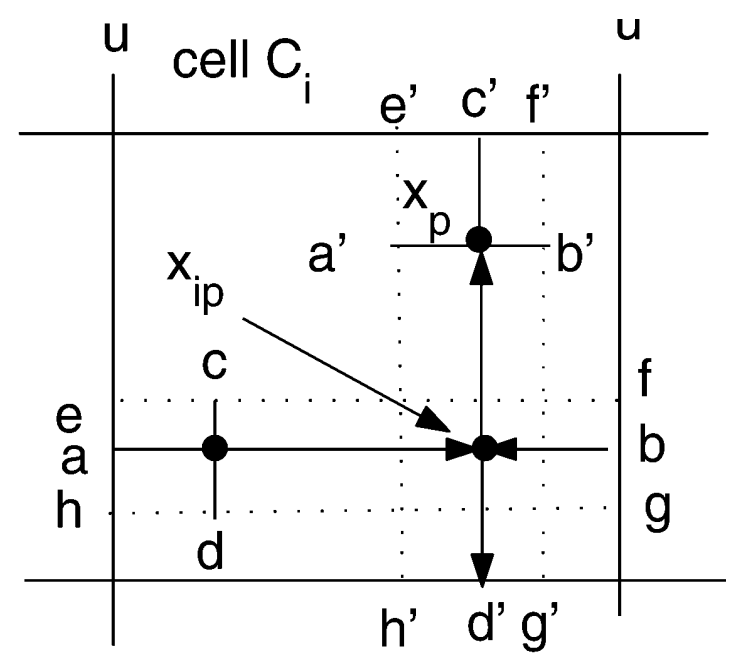

S

Fig. 1. A schematic illustration of a cell in the Markov partition defined with respect to the stable and the unstable manifolds of a hyperbolic chaotic saddle. 
that, at time $p$, the fraction of trajectory points that are still in $B$ is given by

$$
\sum_{\mathbf{x}_{i p} \in C} \frac{1}{L_{1}\left(\mathbf{x}_{i p}\right)},
$$

where the summation is over all unstable fixed points contained in $C$. Since $p<n$, we have

$$
N_{m}(\rho, n, C)=N_{0} \sum_{\mathbf{x}_{i p} \in C} \frac{1}{L_{1}\left(\mathbf{x}_{i p}\right)},
$$

where $\rho=p / n<1$. The natural measure of the chaotic saddle contained in $C$ is thus given by

$$
\begin{aligned}
\mu(C) & =\lim _{n \rightarrow+\infty} \lim _{N_{0} \rightarrow \infty} \frac{N_{m}(\rho, n, C)}{N(n)} \\
& =\lim _{p \rightarrow \infty} \sum_{\mathbf{x}_{i p} \in C} \frac{\exp (p / \tau)}{L_{1}\left(\mathbf{x}_{i p}\right)} .
\end{aligned}
$$

Since $S$ is a phase-space region that contains the whole chaotic saddle, we have $\mu(S)=1$, which, from Eq. (9), implies [Kadanoff \& Tang, 1984]

$$
\mu_{S}(p) \equiv \lim _{p \rightarrow \infty} \sum_{\mathbf{x}_{i p} \in S} \frac{1}{L_{1}\left(\mathbf{x}_{i p}\right)}=\exp (-p / \tau) .
$$

The arguments leading to Eqs. (9) and (10) apply to situations where a proper partition of the phase space exists such that the shorter line segments $a^{\prime} b^{\prime}$ and $c d$ in Fig. 1 are completely contained in the cell $C_{i}$. For hyperbolic systems, such a partition exists, which is the Markov partition [Bowen, 1978]. Therefore, Eqs. (9) and (10) are rigorously valid only for hyperbolic dynamical systems [Grebogi et al., 1988]. Chaotic saddles arising in physical situations are often nonhyperbolic [Lai et al., 1993]. For a nonhyperbolic chaotic saddle, there exists a set of infinite number of points at which the stable and unstable directions coincide. This nonhyperbolicity prevents construction of a grid of cells that look like the one in Fig. 1. Due to this difficulty, there is no rigorous verification of the validity of Eqs. (9) and (10) for nonhyperbolic chaotic saddles. The applicability of Eqs. (9) and (10) to chaotic saddles in nonhyperbolic physical systems thus remains to be a conjecture.

\section{Numerical Verification}

\subsection{Hénon map}

In order to be able to test the applicability of Eqs. (9) and (10) to nonhyperbolic chaotic systems, it is necessary to choose models for which all unstable periodic orbits of up to reasonably high periods

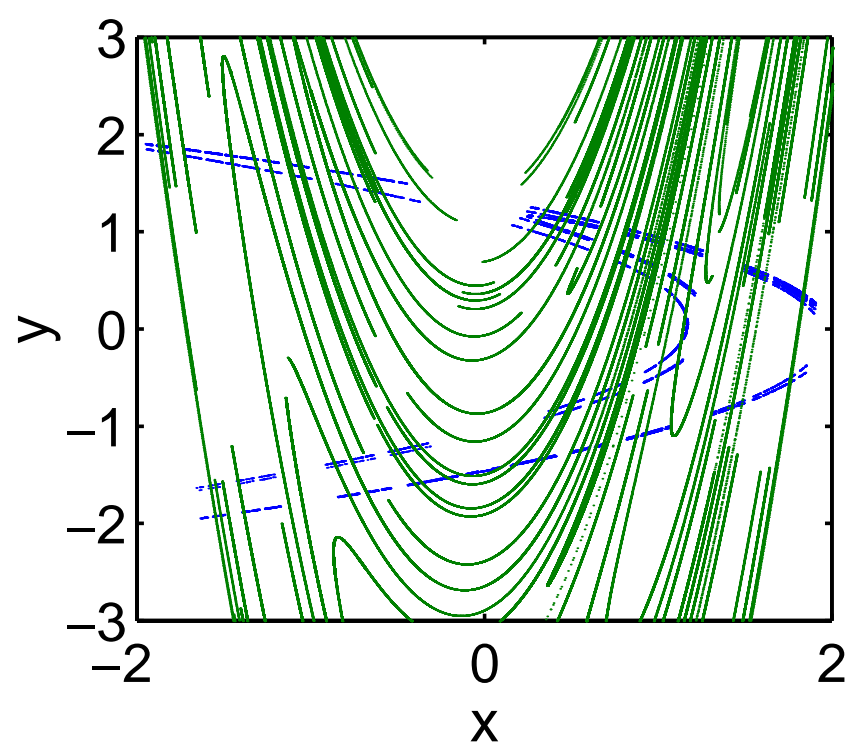

(b)

Fig. 2. (a) A trajectory of $2 \times 10^{4}$ points on the chaotic saddle for the Hénon map at $a=1.6$ and $b=0.3$. The trajectory is computed by using the PIM-triple method. (b) The stable and the unstable foliations of the chaotic saddle in (a), computed by using the sprinkler method. 
can be enumerated. We first choose the Hénon map [Hénon, 1976],

$$
\begin{aligned}
& x_{n+1}=a-x_{n}^{2}+b y_{n}, \\
& y_{n+1}=x_{n},
\end{aligned}
$$

where $a$ and $b$ are parameters. To obtain chaotic saddles, we fix $b=0.3$ and choose $a>a_{c}$, where $a_{c} \approx 1.426$ is the crisis value beyond which the Hénon chaotic attractor is converted into a chaotic saddle [Grebogi et al., 1983]. For $a \gtrsim a_{c}$, explicit numerical computation reveals that the minimally possible angles between the stable and unstable directions for points on the chaotic saddles can be arbitrarily close to zero, indicating that the chaotic saddles are nonhyperbolic [Lai et al., 1993]. Figure 2(a) shows an approximate trajectory, computed by using the PIM-triple method [Nusse \&
Yorke, 1989], of $2 \times 10^{4}$ points on the chaotic saddle for $a=1.6$, and Fig. 2(b) shows the stable and unstable manifolds of the chaotic saddle, which are computed using the sprinkler method [Hsu et al., 1988; Grassberger et al., 1989]. From Fig. 2(b), we see there are apparently points on the chaotic saddle at which the stable and the unstable manifolds tend to coincide. In the sequel, we choose $a=1.5,1.55,1.6$ and 1.65 , for which the chaotic saddles are numerically determined to be nonhyperbolic [Lai et al., 1993], to verify the applicability of Eqs. (9) and (10).

\subsubsection{Validity of Eq. (10)}

We compute all unstable periodic orbits of periods up to 28 and their eigenvalues for $a=1.5,1.55,1.6$ and 1.65. The quantity $\mu_{S}(p)$ in Eq. (10) is then

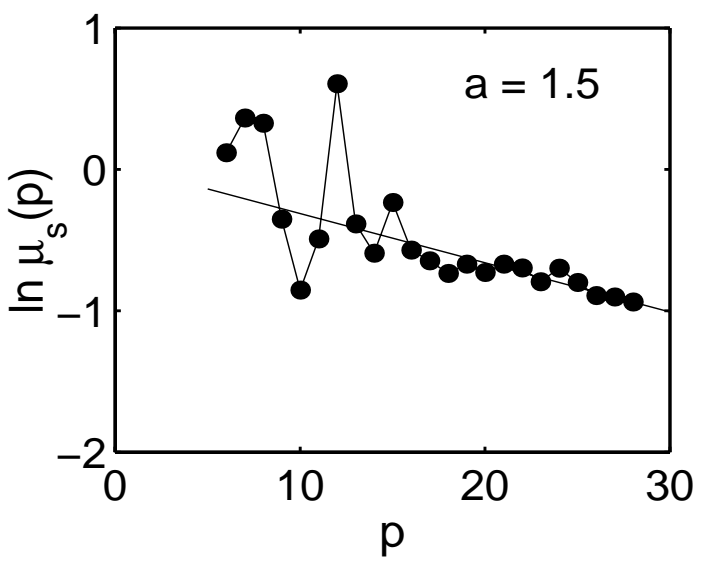

(a)

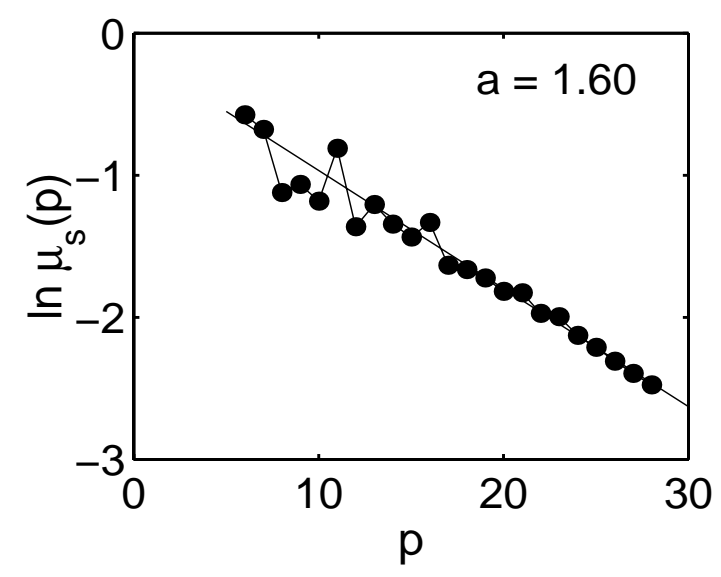

(c)

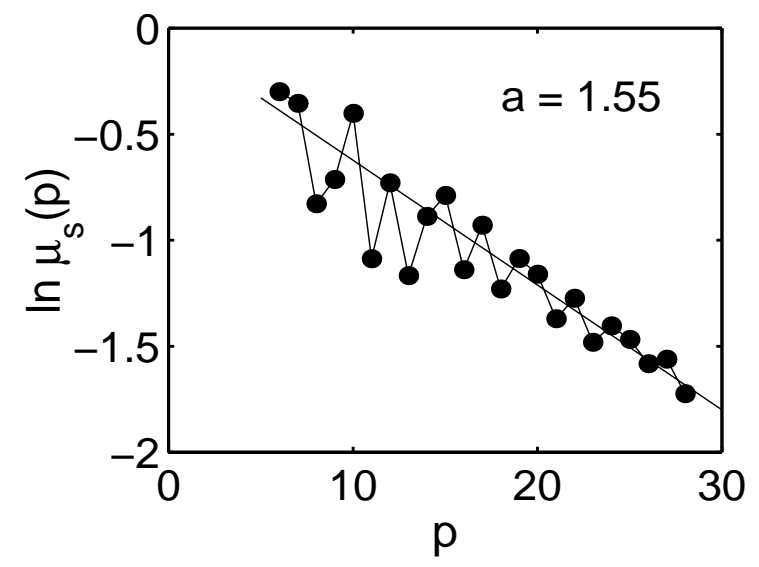

(b)

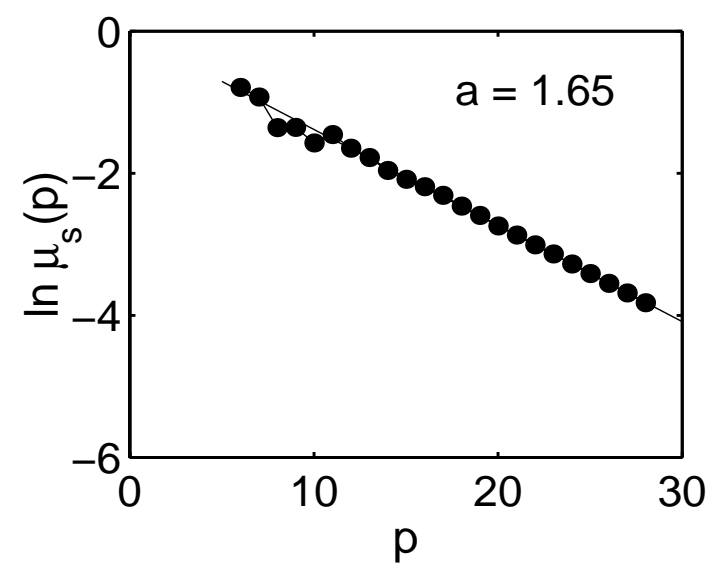

(d)

Fig. 3. (a-d) For the Hénon map at $a=1.5,1.55,1.6$ and 1.65 , respectively, $\ln \mu_{S}(p)$ versus $p$, where $\mu_{S}(p)$ is the total measure represented by all periodic orbits of period $p$ defined in Eq. (10). 


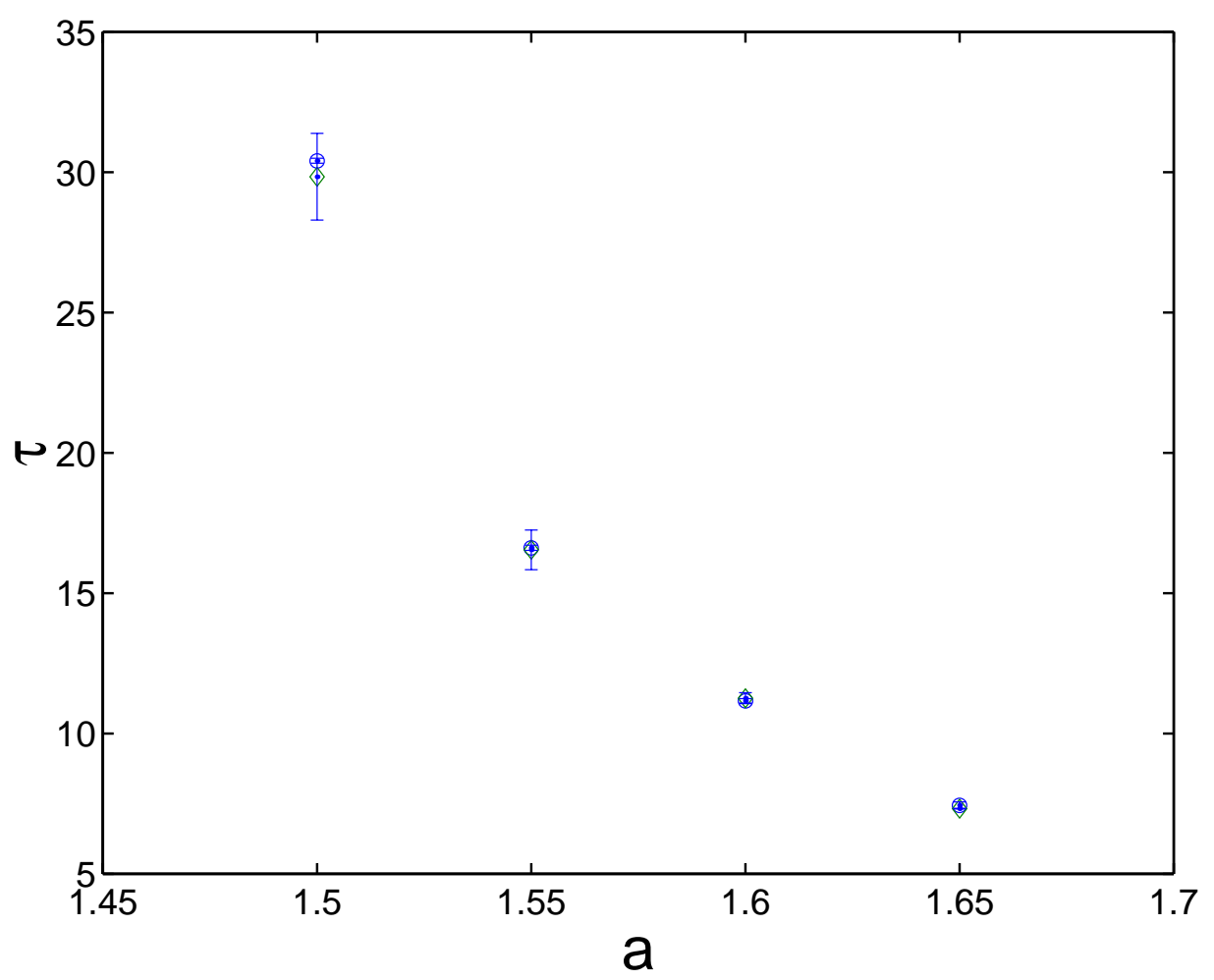

Fig. 4. Comparison of the lifetimes of chaotic saddle obtained by extracting the slopes of the lines of $\ln N(n)$ versus $n$ (circles) and these obtained via Eq. (10) through unstable periodic orbits (diamonds) for $a=1.5,1.55,1.6$ and 1.65 .

computed as a function of $p$, for all periodic orbits of period $p$. Figures $3(\mathrm{a})-3(\mathrm{~d})$ show the results of the computations, where the curves of $\ln \mu_{S}(p)$ versus $p$ are plotted. In all four cases, we observe that $\mu_{S}(p)$ 's decay exponentially, as predicted by Eq. (10). The fitted straight lines in Figs. 3(a)$3(\mathrm{~d})$ indicate the slope $\kappa \equiv 1 / \tau$. To compute $\tau$ from direct numerical realization of Eq. (1), for each $a$ value, we choose $10^{4}$ initial conditions uniformly distributed in the region $-2 \leq(x, y) \leq 2$ and examine, at iteration $n$, the number of trajectory points $N(n)$ that still remain in the region. The lifetimes of the chaotic saddles are then obtained by extracting the slopes of the lines of $\ln N(n)$ versus $n$. Figure 4 shows the lifetimes $\tau$ obtained via this direct approach (circles) and via Eq. (10) through unstable periodic orbits (diamonds) for all four chaotic saddles that we have examined. The closeness of the values of $\tau$ obtained via two different approaches indicates the applicability of Eq. (10) for nonhyperbolic chaotic saddles.

\subsubsection{Validity of Eq. (9)}

To test Eq. (9), we divide the phase-space region: $-2 \leq(x, y) \leq 2$ in which the chaotic saddles lie by using a grid of $128 \times 128$. We use a PIMtriple trajectory of $10^{8}$ points to identify nonempty cells to which the trajectory visits and to compute the frequency of visit, or the approximate natural measure, in each nonempty cell. Call this natural measure $\mu_{i}, i=1, \ldots, N_{n e}$, where $N_{n e}$ is the number of nonempty cells. We then compute, for each nonempty cell $C$, the contribution to the natural measure, $\mu_{i}(p)$, by all periodic orbits of period $p$ contained in the cell. Figures $5(\mathrm{a})-5(\mathrm{~d})$ show $\ln \Delta \mu(p)$ versus $p$ for $a=1.5,1.55,1.6$ and 1.65, respectively, where

$$
\Delta \mu(p) \equiv \frac{1}{N_{n e}} \sum_{i=1}^{N_{n e}}\left|\mu_{i}-\mu_{i}(p)\right| .
$$

We see that $\Delta \mu(p)$ decreases exponentially as $p$ increases, indicating the validity of Eq. (9) for large periods.

We note that Eq. (9) implies that the statistical averages of dynamical invariants and physical functions with respect to the natural measure of the chaotic saddle can be computed in terms of the unstable periodic orbits embedded in the saddle. This offers an alternative way to check the validity of Eq. (9). Specifically, let $F(x, y)$ be a function of 


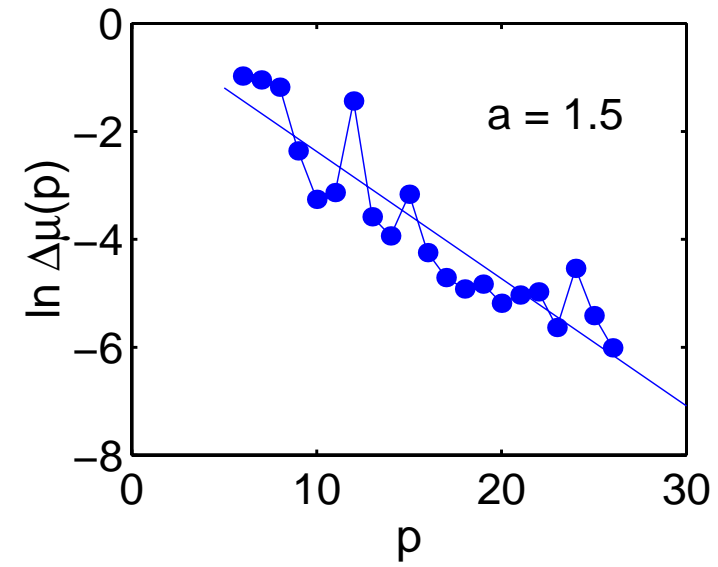

(a)

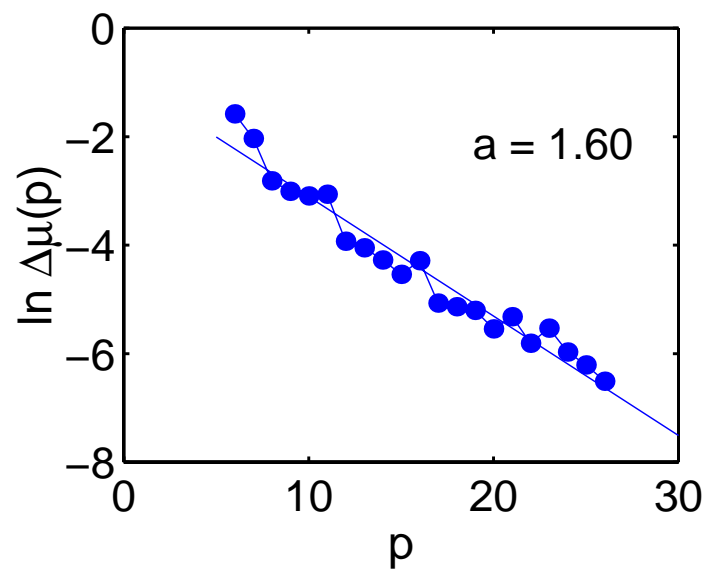

(c)

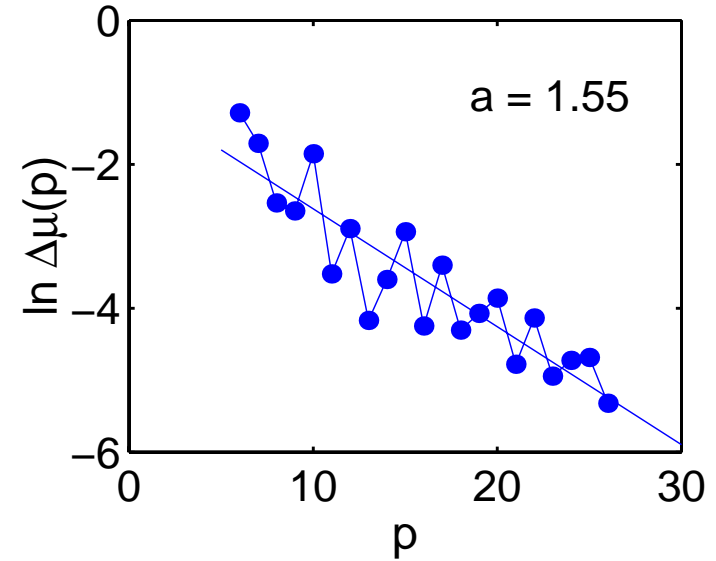

(b)

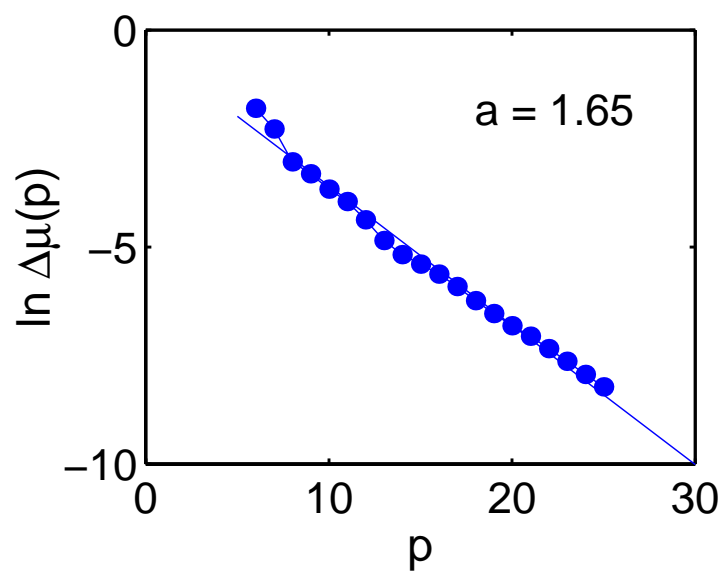

(d)

Fig. 5. (a-d) For the Hénon map at $a=1.5,1.55,1.6$ and 1.65, respectively, $\ln \Delta \mu(p)$ versus $p$. We see that $\Delta \mu(p)$ decreases exponentially as $p$ increases, indicating the applicability of Eq. (9) for large periods.

physical interest. We wish to obtain the average $\langle F\rangle$ of $F(x, y)$ with respect to the natural measure of the chaotic saddle, as follows:

$$
\begin{aligned}
\langle F\rangle & =\int F(x, y) d \mu \\
& =\lim _{N \rightarrow \infty} \frac{1}{N} \sum_{n=1}^{N} F\left(x_{n}, y_{n}\right),
\end{aligned}
$$

where $\left\{x_{n}, y_{n}\right\}(n=1, \ldots, N \rightarrow \infty)$ is a dense trajectory generating the natural measure of the chaotic saddle. In [Nusse \& Yorke, 1989] and [Jacobs et al., 1997], it was argued that the PIMtriple algorithm is capable of yielding trajectories that generate the natural measure of the chaotic saddle. According to Eq. (9), the same average can be expressed in terms of the unstable periodic orbits embedded in the chaotic saddle,

$$
\langle F\rangle(p)=\sum_{j=1}^{N(p)} \mu_{j}(p)\left[\frac{1}{p} \sum_{i=1}^{p} F\left(x_{j i}, y_{j i}\right)\right],
$$

where $\left(x_{j i}, y_{j i}\right)(i=1, \ldots, p)$ denotes the $j$ th fixed point of the $p$-times iterated map, $N(p) \sim e^{h_{T} p}$ is the total number of fixed points of the $p$-times iterated map, and $h_{T}>0$ is the topological entropy of the chaotic saddle. Note that if $F\left(x_{j i}, y_{j i}\right)$ is $\lambda_{j}(p)$, the expanding eigenvalue of the $j$ th fixed point, Eq. (14) yields a representation of the positive Lyapunov exponent of the chaotic saddle in terms of unstable periodic orbits

$$
\lambda(p)=\sum_{j=1}^{N(p)} \mu_{j}(p) \lambda_{j}(p) .
$$




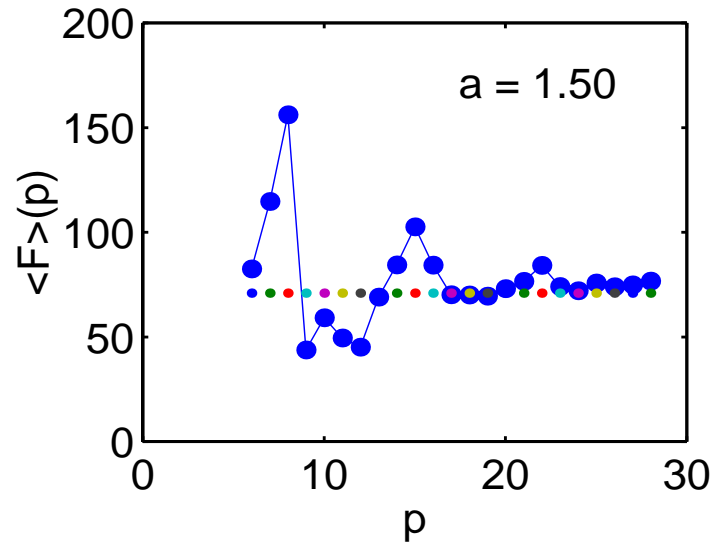

(a)

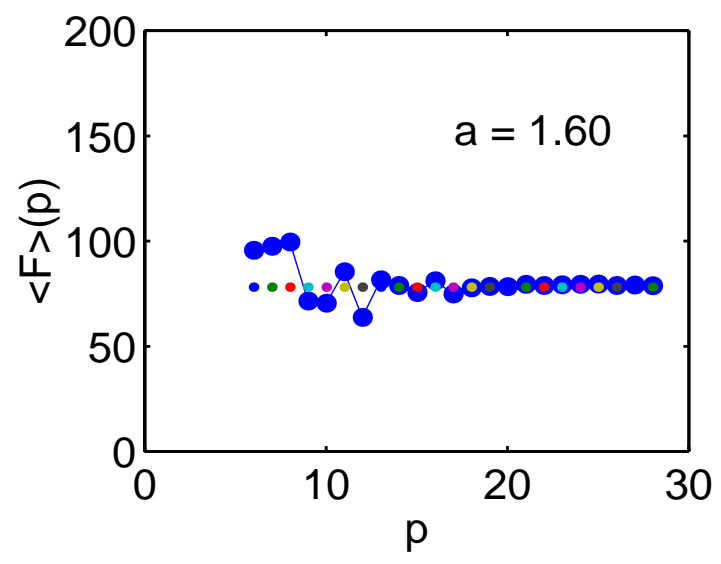

(c)

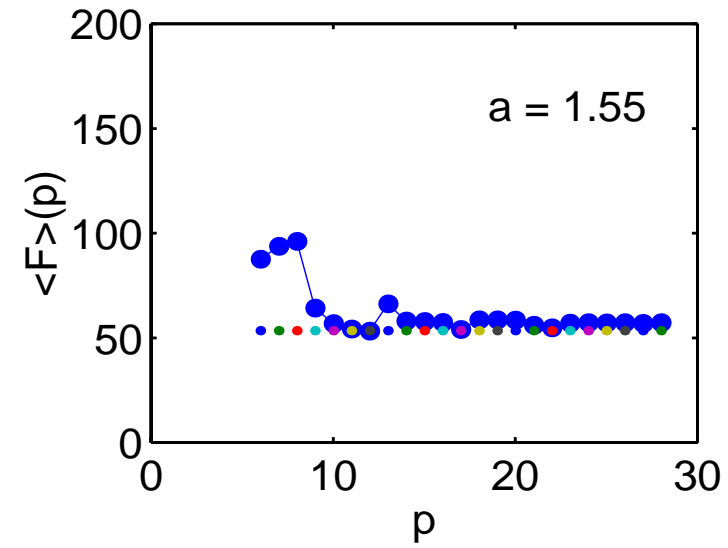

(b)

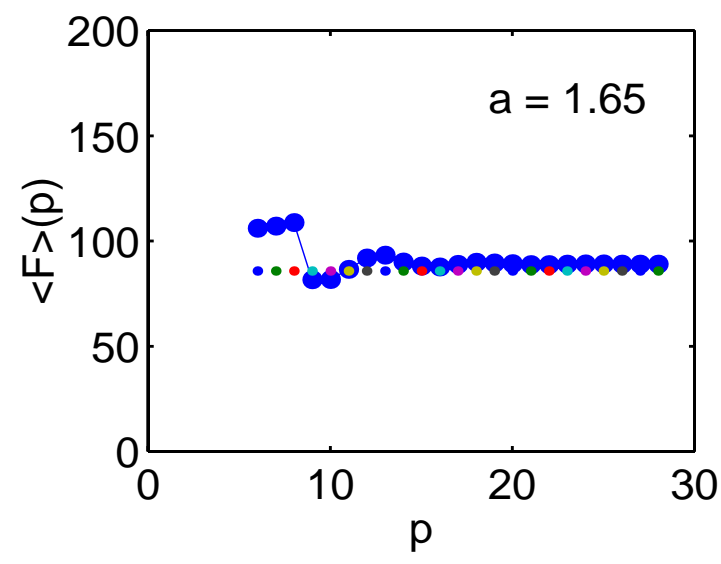

(d)

Fig. 6. (a-d) For the Hénon map at $a=1.5,1.55,1.6$ and 1.65, respectively, $\langle F\rangle$ (dotted line) and $\langle F(p)\rangle$ (large dots) versus $p$, where $F(x, y)=e^{x^{2}+y^{2}}$. It shows that $\langle F(p)\rangle$ converges to $\langle F\rangle$ as $p$ is increased.

Since $\Delta \mu(p)$ decreases exponentially as a function of $p$, we expect the difference $\Delta F(p) \equiv|\langle F\rangle(p)-\langle F\rangle|$ to decrease rapidly as $p$ is increased. Note that for $p$ large but finite, the total measure from all periodic orbits of period $p$ is not exactly equal to but only close to unity. Thus, to evaluate $\langle F\rangle(p)$, we normalize the natural measure $\mu_{j}(p)$ in Eq. (14) by the total measure:

$$
\begin{aligned}
\mu_{j}(p) & =\frac{e^{p / \tau} / L_{1}\left(\mathbf{x}_{j p}, p\right)}{\sum_{i=1}^{N(p)} e^{p / \tau} / L_{1}\left(\mathbf{x}_{i p}, p\right)} \\
= & \frac{1 / L_{1}\left(\mathbf{x}_{j p}, p\right)}{\sum_{i=1}^{N(p)} 1 / L_{1}\left(\mathbf{x}_{i p}, p\right)}, \quad j=1, \ldots, N(p) .
\end{aligned}
$$

We first consider a smooth function: $F(x, y)=$ $e^{x^{2}+y^{2}}$. Its average with respect to the natural measure of the chaotic saddle is computed by using $10^{4}$ PIM-triple trajectories, each having length 1000 with 1000 preiterations. We then compute $\langle F\rangle(p)$ from Eq. (14) by using periodic orbits of period up to 28. Figures $6(\mathrm{a})-6(\mathrm{~d})$ show $\langle F\rangle(p)$ versus $p$ for the Hénon map for $a=1.5,1.55,1.6$ and 1.65, respectively. In all four cases, we observe that $\langle F\rangle(p)$ rapidly approaches $\langle F\rangle$ as $p$ becomes large.

Next, we compute the positive Lyapunov exponent of the chaotic saddle by using: (i) a long PIM-triple trajectory; and (ii) periodic orbits as in Eq. (15). Figures $7(\mathrm{a})-7(\mathrm{~d})$ show $\lambda(p)$ versus $p$ for $a=1.5,1.55,1.6$ and 1.65, respectively. We see that, again, $\lambda(p)$ approaches $\lambda$ as $p$ is increased. These results suggest that the characterization of the natural measure of nonhyperbolic chaotic saddles by unstable periodic orbits becomes more precise as the period is increased. 


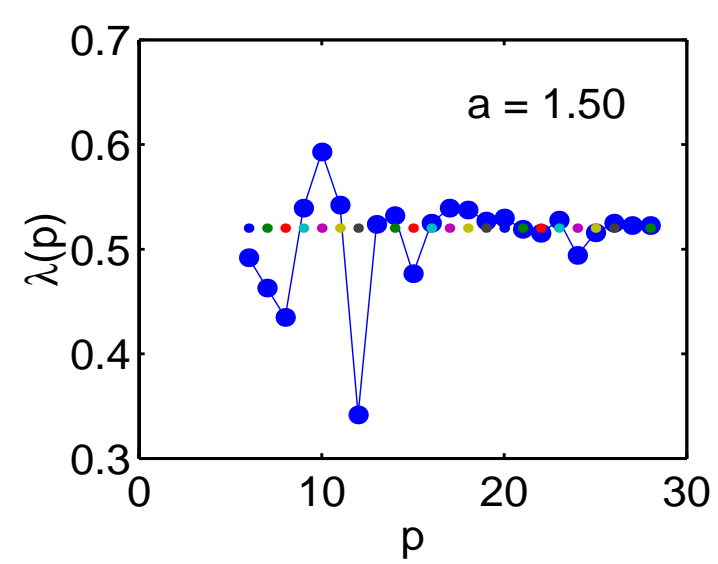

(a)

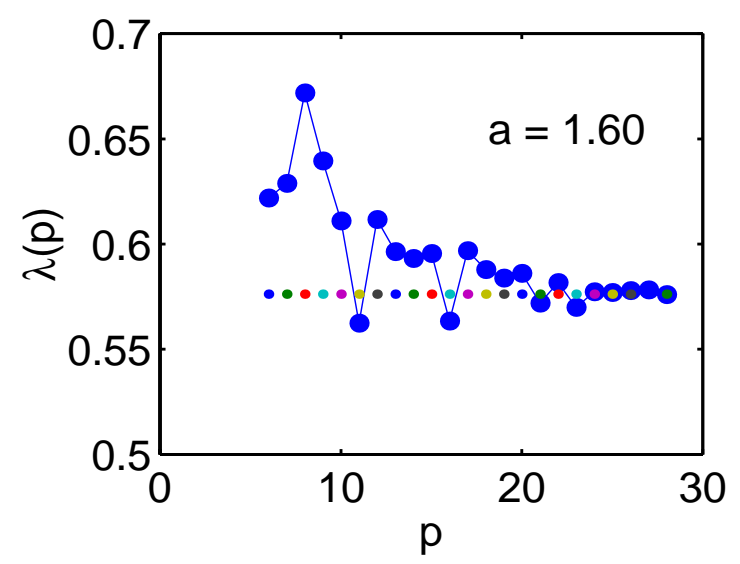

(c)

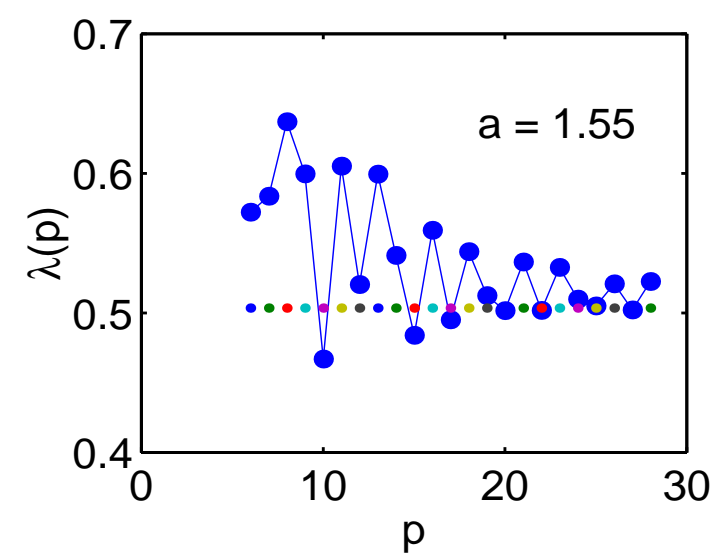

(b)

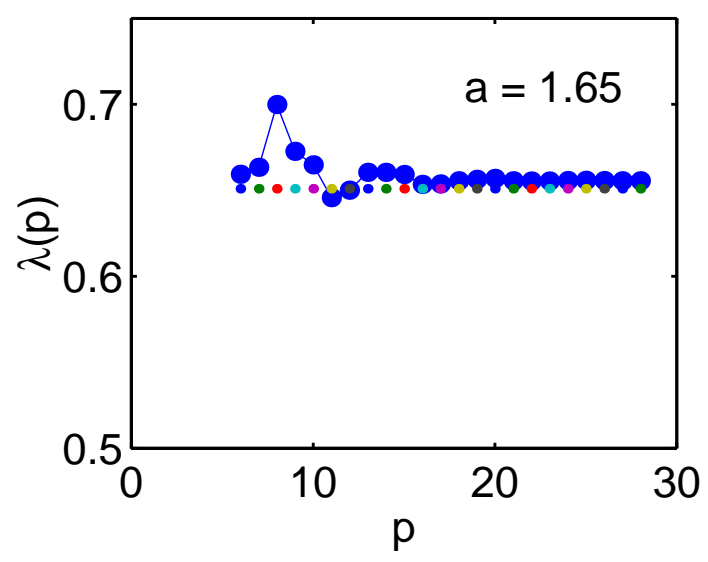

(d)

Fig. 7. (a-d) For the Hénon map at $a=1.5,1.55,1.6$ and 1.65, respectively, $\lambda$ (dotted line) and $\lambda(p)$ (large dots) versus $p$, where $\lambda$ and $\lambda(p)$ are the positive Lyapunov exponent of the chaotic saddle computed from the natural measure and from all periodic orbits of period $p$, respectively.

\subsection{Ikeda-Hammel-Jones-Moloney map}

To provide further numerical support for the validity of Eqs. (9) and (10), we now consider the following Ikeda-Hammel-Jones-Moloney (IHJM) map [Ikeda, 1979; Hammel et al., 1985]:

$$
\begin{aligned}
& x_{n+1}=a+b\left(x_{n} \cos \phi_{n}-y_{n} \sin \phi_{n}\right), \\
& y_{n+1}=b\left(x_{n} \sin \phi_{n}+y_{n} \cos \phi_{n}\right),
\end{aligned}
$$

where $\phi_{n}=k-\eta /\left(1+x_{n}^{2}+y_{n}^{2}\right)$ is the phase variable, $a, b, k$ and $\eta$ are parameters. The IHJM map models the dynamics of an optical pulse propagating in a ring cavity, subject to partial reflection, phase and amplitude modulation and distortion due to a nonlinear optical medium in the cavity. Specifically, the optical field is represented by the complex variable $z=x+i y$, the parameters $a$ and $b$ quantify the splitting of the optical field at various mirrors in the cavity, the term $\eta /\left(1+x_{n}^{2}+y_{n}^{2}\right)$ simulates the phase modulation due to the nonlinear medium, and the parameter $k$ characterizes the optical detuning of the cavity in the absence of nonlinear medium. In our numerical experiments, we fix $b=0.9, k=0.4$, $\eta=6.0$ and choose $a$ as the bifurcation parameter. We observe that a boundary crisis [Grebogi et al., $1983]$ occurs at $a_{c} \approx 1.01$, where a chaotic attractor for $a \lesssim a_{c}$ is converted into a chaotic saddle for $a \gtrsim a_{c}$. Figure 8(a) shows, for $a=1.1$, a PIMtriple trajectory of the chaotic saddle. The chaotic saddle is apparently nonhyperbolic, as tangencies between the stable manifold (trace of gaps along the chaotic saddle) and the unstable manifold (the closure of the chaotic saddle) can even be visually seen from Fig. 8(a).

In order to test the validity of Eq. (10) for the chaotic saddle in Fig. 8, we compute all unstable 


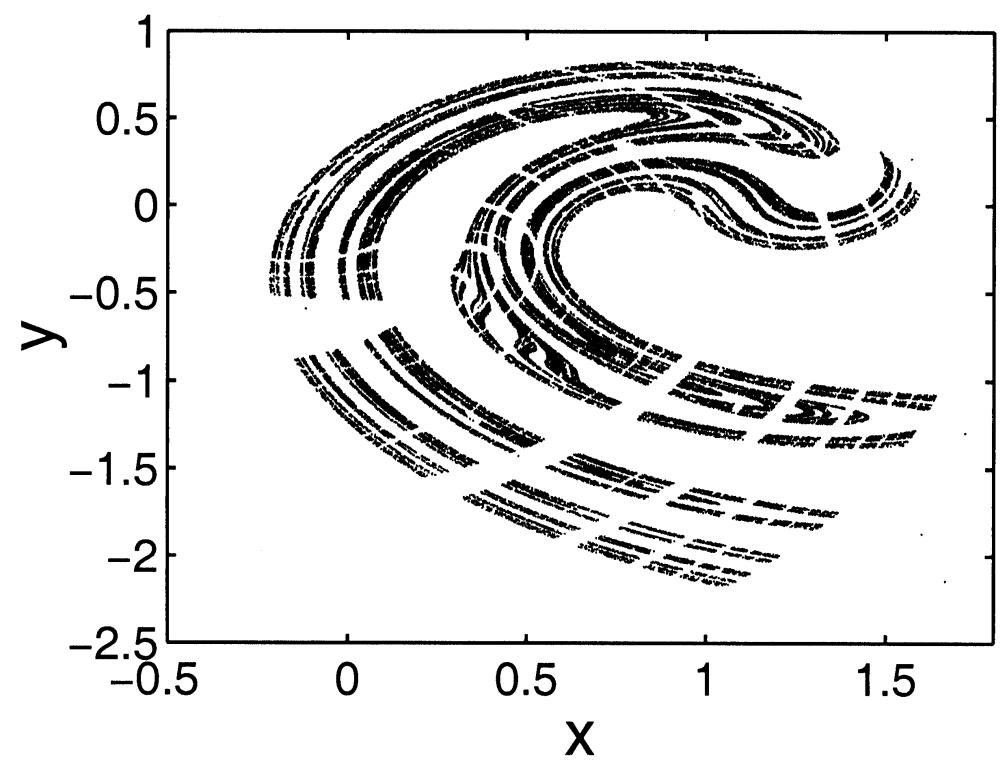

(a)

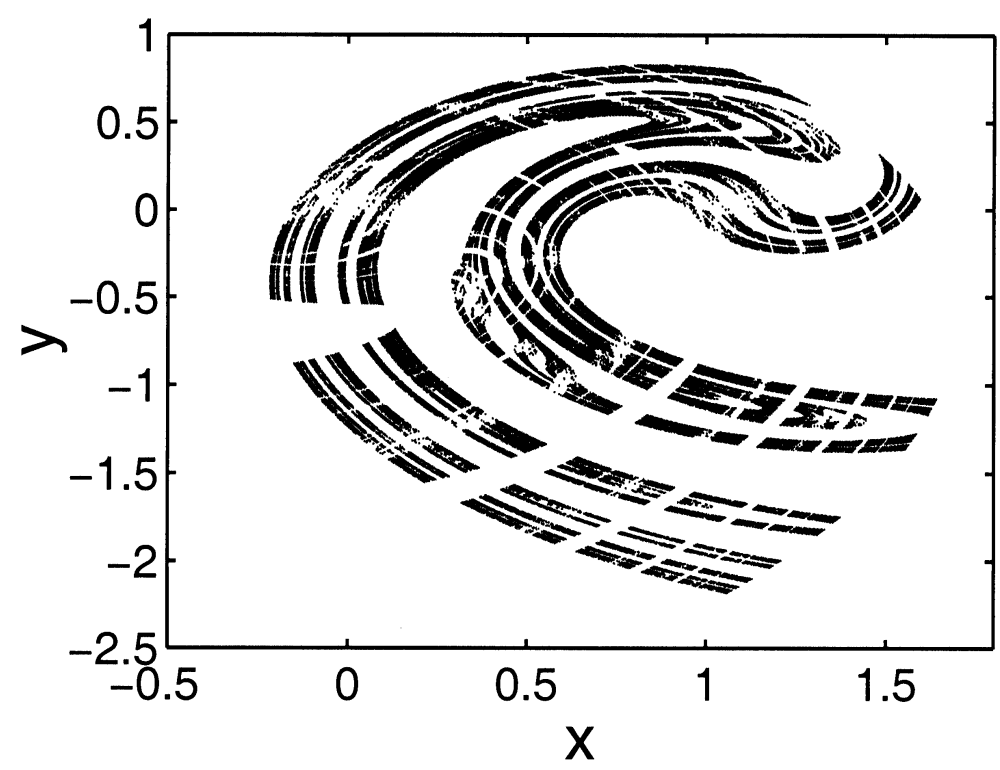

(b)

Fig. 8. (a) A trajectory of $9 \times 10^{4}$ points on the chaotic saddle for the IHJM map at $a=1.1, b=0.9, k=0.4$ and $\eta=6.0$. The trajectory is computed by using the PIM-triple method. (b) Locations of 343,094 orbit points of all periodic orbits of periods 20 and their factors.

periodic orbits of periods up to 20 by using the algorithm recently developed by Davidchack and Lai [1999]. Figure 8(b) shows the locations of 343094 orbit points of all periodic orbits of periods 20 and their factors embedded in the chaotic saddle. We see that, qualitatively, Fig. 8(b) resemble Fig. 8(a), indicating that the natural measure of the chaotic saddle can be represented by unstable periodic orbits of high periods. Figure 9 (a) shows $\ln N(p)$ versus $p$, the slope of which gives the topological entropy of the chaotic saddle in Fig. 8(a). We obtain: $h_{T} \approx 0.64$. Note from Fig. 9 (a) that the linear fit becomes more robust for $p>10$. Thus, we expect the exponential behavior of $\mu_{S}(p)$ in Eq. (10) to be seen for $p>10$, as shown in Fig. 9(b), where $\ln \mu_{S}(p)$ versus $p$, together with 


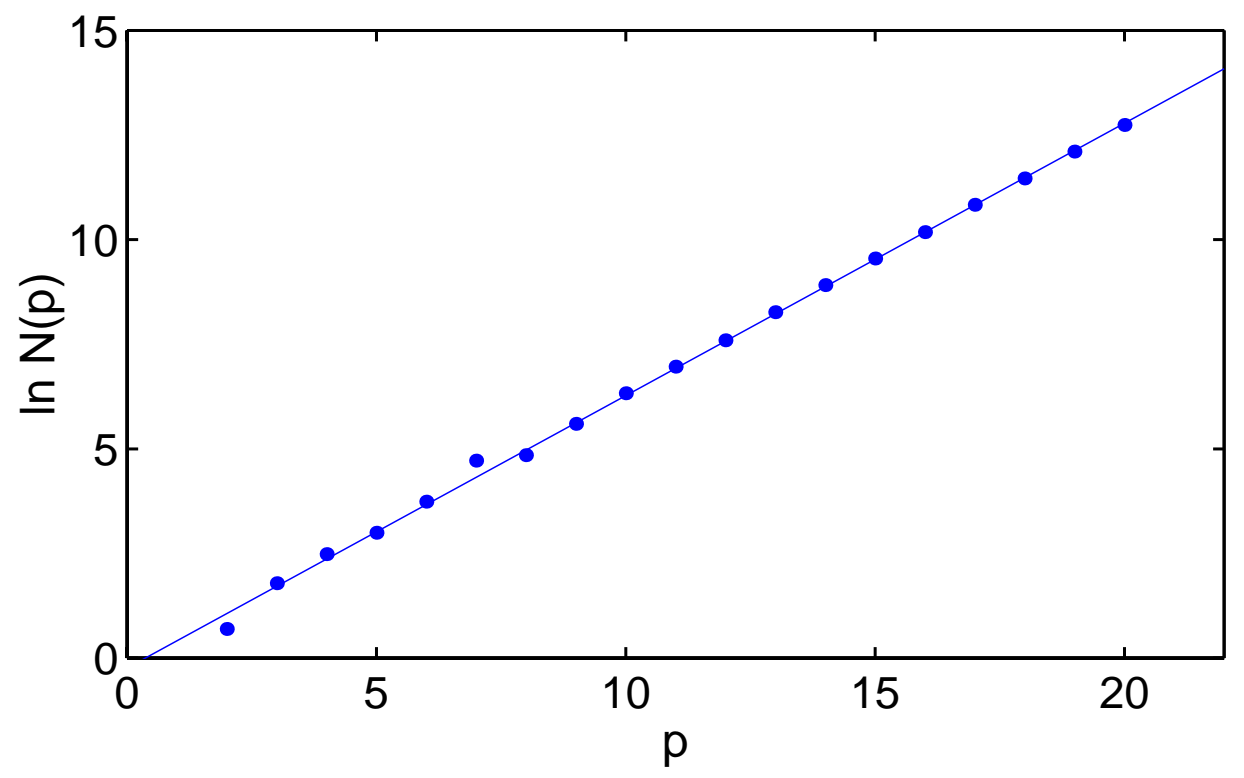

(a)

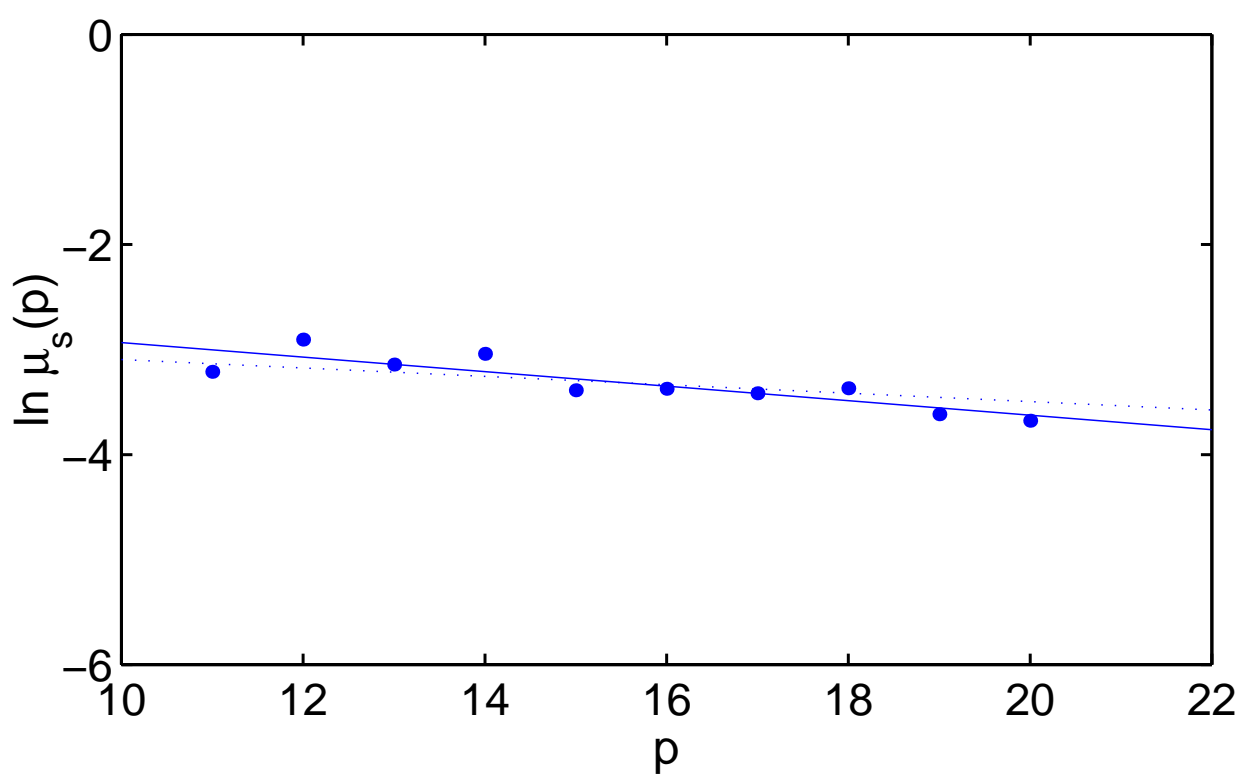

(b)

Fig. 9. For the IHJM map, (a) $\ln N(p)$ versus $p$, the slope of the linear fit gives the topological entropy of the chaotic saddle: $h_{T} \approx 0.64$. (b) $\ln \mu_{S}(p)$ versus $p$, where $\mu_{S}(p)$ is the total measure of all the periodic orbits of period $p$ defined in Eq. (10). The slope of the dotted line represents the escaping rate obtained from direct simulation of Eq. (1).

a linear fit, is plotted, for $11 \leq p \leq 20$. The inverse of the slope of this decay gives the average lifetime of the chaotic saddle in Fig. 8(a). We obtain $\tau \approx 22$. By sprinkling a large number of initial conditions in the region of Fig. 8 and examining how they decay in time, we obtain $\tau \approx 28$ (the inverse of $\tau$ is indicated by the slope of the dashed line in Fig. 9). The discrepancy between this value of $\tau$ and the one obtained from unstable periodic orbits is due to the low periods utilized in Fig. 9, as periodic orbits only up to period 20 are computed. It is, however, computationally demanding to go beyond period 20 because the chaotic saddle in Fig. 8(a) has a relatively large value of topological entropy $\left(h_{T} \approx 0.64\right)$. We have also considered a smooth function: $F(x, y)=e^{x^{2}+y^{2}}$ and computed 


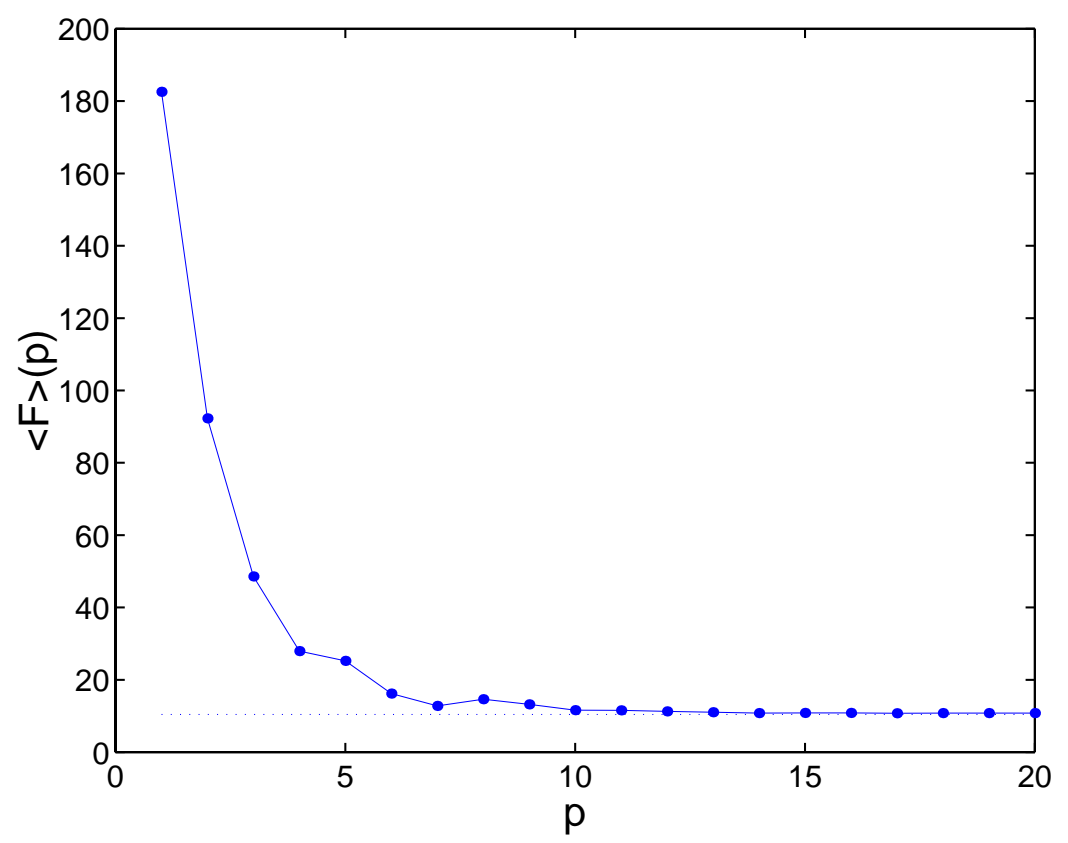

Fig. 10. For the IHJM map, $\langle F\rangle$ (dotted line) and $\langle F(p)\rangle$ (continuous line with dots) versus $p$, where $F(x, y)=e^{x^{2}+y^{2}}$. Again, $\langle F(p)\rangle$ converges to $\langle F\rangle$ as $p$ is increased.

its average, $\langle F\rangle$, with respect to the natural measure of the chaotic saddle in Fig. 8(a), and $\langle F\rangle(p)$ from Eq. (14) by using periodic orbits of period up to 20 . Figure 10 shows $\langle F\rangle(p)$ versus $p$, where we see that as $p$ is increased, $\langle F\rangle(p)$ rapidly approaches $\langle F\rangle$. These results thus indicate the goodness of the unstable periodic orbit characterization of the natural measure for nonhyperbolic chaotic saddles in the IHJM map.

\section{Discussions}

Unstable periodic orbits are believed to be the skeleton of chaotic dynamics [Morita et al., 1987; Gunaratne \& Procaccia, 1987; Auerbach et al., 1988a; Auerbach et al., 1988b; Auerbach, 1990; Cvitanović, 1992; Christiansen et al., 1997]. They are the most fundamental building blocks of classical chaos with usefulness in the study of quantum chaos [Gutzwiller, 1990]. Dynamical invariants of a chaotic system such as the Lyapunov exponents and the fractal dimension can be expressed in terms of properties of unstable periodic orbits, which is theoretically appealing. Since these dynamical invariants are defined with respect to the natural measure, an important step is to relate the natural measure to the set of infinite number of unstable periodic orbits embedded in the chaotic invariant set. Previous works have firmly established the validity of the quantitative characterization of the natural measure for chaotic attractors, hyperbolic or nonhyperbolic [Grebogi et al., 1988; Lai et al., 1997].

The principal contribution of this paper is strong numerical evidence for the applicability of the periodic-orbit theory of the natural measure for another important class of dynamical invariant sets that arise commonly in situations of physical interest: nonattracting chaotic saddles. Our emphasis is on nonhyperbolic chaotic saddles for which the periodic-orbit characterization of the natural measure remains only a conjecture [Grebogi et al., 1988]. Our systematic computations suggest that such a characterization is generally valid for nonhyperbolic chaotic saddles, which are typical in the parameter space of chaotic systems, particularly after the crisis at which the chaotic saddle is born. ${ }^{2}$

\footnotetext{
${ }^{2}$ Suppose we examine whether the chaotic set is nonhyperbolic or hyperbolic as a system parameter is changed. Chaotic attractors arising in physical systems are always nonhyperbolic [Alligood et al., 1997]. Immediately after the crisis at which a chaotic attractor is converted into a chaotic saddle, there can be intervals of parameter values in which the chaotic saddles are nonhyperbolic, although nonhyperbolicity becomes rare in parameter regimes far beyond the crisis value [Lai et al., 1993]. These parameter intervals are the Newhouse intervals [Newhouse, 1979].
} 


\section{Acknowledgments}

This work was sponsored by AFOSR under Grant No. F49620-98-1-0400 and by NSF under Grant No. PHY-9996454.

\section{References}

Alligood, K. T., Sauer, T. \& Yorke, J. A. [1996] Chaos: An Introduction to Dynamical Systems (Springer, NY).

Auerbach, D., Cvitanović, P., Eckmann, J.-P., Gunaratne, G. H. \& Procaccia, I. [1988a] "Exploring chaotic motion through periodic orbits," Phys. Rev. Lett. 58, 2387-2389.

Auerbach, D., O'Shaughnessy, B. \& Procaccia, I. [1988b] "Scaling structure of strange attractors," Phys. Rev. A37, 2234-2236.

Auerbach, D. [1990] "Scaling of periodic orbits in 2-dimensional chaotic systems," Phys. Rev. A41, 6692-6701.

Bowen, R. [1978] "Markov partitions are not smooth," Proc. Am. Math. Soc. 71, 130-132.

Christiansen, F., Cvitanović, P. \& Putkaradze, V. [1997] "Spatiotemporal chaos in terms of unstable recurrent patterns," Nonlinearity 10, 55-70.

Davidchack, R. \& Lai, Y.-C. [1999] "Efficient algorithm for detecting unstable periodic orbits in chaotic systems," Phys. Rev. E60, 6172-6175.

Davidchack, R., Lai, Y.-C., Klebanoff, A. \& Bollt, E. M. [2001] "Towards complete detection of unstable periodic orbits in chaotic systems," Phys. Lett. A287, 99-104.

Dawson, S., Grebogi, C., Sauer, T. \& Yorke, J. A. [1994] "Obstructions to shadowing when a Lyapunov exponent fluctuates about zero," Phys. Rev. Lett. 73, 1927-1930.

Dhamala, M. \& Lai, Y.-C. [1999] "Unstable periodic orbits and the natural measure of nonhyperbolic chaotic saddles," Phys. Rev. E60, 6176-6179.

Eckhardt, B. \& Aref, H. [1988] "Integrable and chaotic motions of 4 vortices. 2. collisions dynamics of vortex pairs," Trans. Soc. Roy. Lond. A326, 655-696.

Focus Issue [1992] Chaos 2, 1-158.

Focus Issue [1993] Chaos 3, 417-482.

Grassberger, P., Kantz, H. \& Moenig, U. [1989] "On the symbolic dynamics of the Hénon map," J. Phys. A: Math. Gen. 22, 5217-5230.

Grebogi, C., Ott, E. \& Yorke, J. A. [1982] "Chaotic attractors in crisis," Phys. Rev. Lett. 48, 1507-1510.

Grebogi, C., Ott, E. \& Yorke, J. A. [1983] "Crises, sudden changes in chaotic attractors and chaotic transients," Physica D7, 181-200.

Grebogi, C., Ott, E. \& Yorke, J. A. [1988] "Unstable periodic orbits and the dimensions of multifractal chaotic attractors," Phys. Rev. A37, 1711-1724.
Grebogi, C., Hammel, S. M., Yorke, J. A. \& Sauer, T. [1990] "Shadowing of physical trajectories in chaotic dynamics: Containment and refinement," Phys. Rev. Lett. 65, 1527-1530.

Gunaratne, G. H. \& Procaccia, I. [1987] "Organization of chaos," Phys. Rev. Lett. 59, 1377-1380.

Gutzwiller, M. C. [1990] Chaos in Classical and Quantum Mechanics (Springer-Verlag, Berlin).

Hammel, S. M., Jones, C. K. R. T. \& Moloney, J. [1985] "Global dynamical behavior of the optical field in a ring cavity," J. Opt. Soc. Am. B2, 552-564.

Hammel, S. M., Yorke, J. A. \& Grebogi, C. [1987] "Do numerical orbits of chaotic dynamical processes represent true orbits?" J. Complexity 3, 136-145.

Hammel, S. M., Yorke, J. A. \& Grebogi, C. [1988] "Numerical orbits of chaotic processes represent true orbits," Bull. Am. Math. Soc. 19, 465-469.

Hénon, M. [1976] "2-Dimensional mapping with a strange attractor," Comm. Math. Phys. 50, 69-77.

Hsu, G.-H, Ott, E. \& Grebogi, C. [1988] "Strange saddles and the dimensions of their invariant-manifolds," Phys. Lett. A127, 199-204.

Ikeda, K. [1979] "Multiple-valued stationary state and its instability of the transmitted light by a ring cavity system," Opt. Commun. 30, 257-261.

Jacobs, J., Ott, E. \& Grebogi, C. [1997] "Computing the measure of nonattracting chaotic sets," Physica D108, $1-11$.

Jung, C., Tél, T. \& Ziemniak, E. [1993] "Application of scattering chaos to particle transport in a hydrodynamic flow," Chaos 3, 555-568.

Kadanoff, L. P. \& Tang, C. [1984] "Escape from strange repellers," Proc. Natl. Acad. Sci. 81, 1276-1279.

Kantz, H. \& Grassberger, P. [1985] "Repellers, semiattractors, and long-lived chaotic transients," Physica D17, 75-86.

Lai, Y.-C., Grebogi, C., Yorke, J. A. \& Kan, I. [1993] "How often are chaotic saddles nonhyperbolic?" Nonlinearity 6, 779-797.

Lai, Y.-C. [1997] "Characterization of the natural measure by unstable periodic orbits in nonhyperbolic chaotic systems," Phys. Rev. E56, 6531-6539.

Lai, Y.-C., Nagai, Y. \& Grebogi, C. [1997] "Characterization of the natural measure by unstable periodic orbits in chaotic attractors," Phys. Rev. Lett. 79, 649-652.

McDonald, S. W., Grebogi, C., Ott, E. \& Yorke, J. A. [1985] "Fractal basin boundaries," Physica D17, $125-153$.

Morita, T., Hata, H., Mori, H., Horita, T. \& Tomita, K. [1987] "On partial dimensions and spectra of singularities of strange attractors," Prog. Theor. Phys. 78, 511-515.

Neufeld, Z. \& Tél, T. [1998] "Advection in chaotically time-dependent open flows," Phys. Rev. E57, 2832-2842.

Newhouse, S. E. [1979] "The abundance of wild hyper- 
bolic sets and nonsmooth sets for diffeomorphisms," Publ. Math. IHES 50, 101-151.

Nusse, H. E. \& Yorke, J. A. [1989] "A procedure for finding numerical trajectories on chaotic saddles," Physica D36, 137-156.

Péntek, Á., Tél, T. \& Toroczkai, Z. [1995a] "Chaotic advection in the velocity-field of leapfrogging vortex pairs," J. Phys. A28, 2191-2216.

Péntek, Á., Tél, T. \& Toroczkai, Z. [1995b] "Fractal tracer patterns in open hydrodynamical flows - the case of leapfrogging vortex pairs," Fractals 3, 33-53.

Péntek, Á., Toroczkai, Z., Tél, T., Grebogi, C. \& Yorke, J. A. [1995c] "Fractal boundaries in open hydrodynamical flows - signatures of chaotic saddles," Phys. Rev. E51, 4076-4088.

Smale, S. [1967] "Differentiable dynamical systems," Bull. Amer. Math. Soc. 73, 747-817.

Szabó, K. G., Lai, Y.-C., Tél, T. \& Grebogi, C. [1996] "Critical exponent for gap filling at crisis," Phys. Rev. Lett. 77, 3102-3105.

Szabó, K. G., Lai, Y.-C., Tél, T. \& Grebogi, C. [2000] "Topological scaling and gap filling at crisis," Phys. Rev. E51, 5019-5032.

Tél, T. [1990] Transient Chaos, Directions in Chaos, Vol. 3, ed. Hao, B.-L. (World Scientific, Singapore), pp. 149-221.

Tél, T. [1996] "Transient chaos: A type of metastable state," STATPHYS 19, ed. Hao, B.-L. (World Scientific, Singapore), pp. 346-362.

Toroczkai, Z., Károlyi, G., Péntek, Á., Tél, T. \& Grebogi, C. [1998] "Advection of active particles in open chaotic flows," Phys. Rev. Lett. 80, 500-503.

Ziemniak, E., Jung, C. \& Tél, T. [1994] "Application of scattering chaos to particle transport in a hydrodynamic flow," Physica D76, 123-146.

\section{Appendix Computation of Unstable Periodic Orbits}

Consider an $N$-dimensional chaotic map: $\mathbf{x}_{n+1}=$ $\mathbf{f}\left(\mathbf{x}_{n}\right)$. The orbit points of period $p$ can be detected as the zeros of the following function: $\mathbf{g}(\mathbf{x})=$ $\mathbf{f}^{(p)}(\mathbf{x})-\mathbf{x}$, where $\mathbf{f}^{(p)}(\mathbf{x})$ is the $p$-times iterated map of $\mathbf{f}(\mathbf{x})$. To find zeros of $\mathbf{g}(\mathbf{x})$, one usually chooses an initial point $\mathbf{x}_{0}$ and then computes successive corrections: $\mathbf{x}_{k+1}=\mathbf{x}_{k}+\delta \mathbf{x}$, which converge to the desired solution. We use the following iterative scheme [Davidchack \& Lai, 1999; Davidchack et al., 2001]:

$$
\mathbf{x}_{k+1}=\mathbf{x}_{k}+[\mathbf{1} \beta g(\mathbf{x})-\mathbf{C J}(\mathbf{x})]^{-1} \cdot \mathbf{C g}(\mathbf{x}),
$$

where $g(\mathbf{x}) \equiv\|\mathbf{g}(\mathbf{x})\| \geq 0$ is the length of the vector, and $\beta>0$ is an adjustable parameter. In the vicinity of an UPO, the function $g(\mathbf{x})$ tends to zero so the scheme becomes the traditional Newton-Raphson scheme with fast convergence. Away from the solution and for sufficiently large values of $\beta$, the scheme has the property of global convergence [Davidchack \& Lai, 1999; Davidchack et al., 2001]. The scheme is in fact the semi-implicit Euler method with step size $h=1 / \beta g(\mathbf{x})$ for solving the same system of ODEs. Numerically, the procedure consists of the following steps:

1. Find all periodic orbits of low periods, say, all fixed points and all period-2 orbits, by using any of the described above iteration schemes with a sufficiently large number of randomly chosen seeds;

2. List all $2^{N} N$ ! matrices $\mathbf{C}_{N \times N}$, and determine the subset of these necessary for stabilizing arbitrary hyperbolic equilibria;

3. Iterate Eq. (A.1) by using every periodic point of period $p-1$ as seeds, and choosing a matrix $\mathbf{C}$ and a number $\beta=\beta_{1}>0$;

4. If the sequence $\left\{\mathbf{x}_{k}\right\}$ converges to a root of $\mathbf{g}$, then iterate $\mathbf{f}$ to find all components of the orbit;

5. Repeat steps (3) and (4) for every matrix $\mathbf{C}$ in the list;

6. Repeat steps (3)-(5) for increasing values of $\beta$ until no more new orbit points are found. 